\section{Revista de la \\ Universidad del Thulia}

Fundada en 1947 por el Dr. Jesúns Enrique Lossada

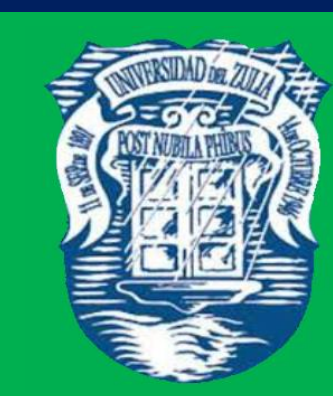

Ciencias del

Algreo

Ingemieria

y Teemología

\section{Aกัต 11 No 29}

Enero - Abril 2021

Tercera Época

Maracaibo-Venezuela 
REVISTA DE LA UNIVERSIDAD DEL ZULIA. $3^{a}$ época. Año 11 № 29, 2020

Rafael Galíndez et al./// Diversidad fenotípica de aves criollas de postura...412-427

\title{
Diversidad fenotípica de aves criollas de postura basada en caracteres zoométricos
}

\author{
Rafael Galíndez * \\ Glenn Lucas* \\ Omar Colmenares **
}

RESUMEN

Con la finalidad de analizar la diversidad fenotípica de aves criollas venezolanas de postura del Laboratorio Sección de Aves, Instituto de Producción Animal, Facultad de Agronomía, Universidad Central de Venezuela, se tomaron medidas zoométricas de 220 gallos y gallinas de las razas FAGRO $(\mathrm{n}=33)$, GDB $(\mathrm{n}=65)$, IPA $(\mathrm{n}=36)$ y Maracay $(\mathrm{n}=86)$. Se consideró el peso vivo, perímetro torácico, longitud corporal, muslo, pierna, tarso, ala, orejilla, barbilla, plumas de cola, altura de cresta y ancho de orejilla. Se realizaron análisis de varianza incluyendo los efectos de sexo y raza, comparando los promedios con la prueba de Duncan. Se calcularon los coeficientes de correlación de algunas medidas corporales. Se ejecutó un análisis discriminante, se calcularon las distancias de Mahalanobis y se graficaron los centroides de cada raza. Todas las medidas resultaron superiores $(\mathrm{P}<0,05)$ en los machos con diferencias entre 1 y $57 \%$. Se detectaron divergencias $(\mathrm{P}<0,05)$ entre razas, siendo Maracay e IPA las que expresaron mayores valores. La mayoría de las correlaciones de las medidas corporales resultaron altamente significativas, observándose magnitudes superiores en la raza IPA. Las distancias de Mahalanobis basadas en seis caracteres discriminantes, revelaron que las razas más alejadas son Maracay y FAGRO. Se concluye que existe marcado dimorfismo sexual en las aves estudiadas; asimismo, se detectó alta diversidad entre los grupos analizados.

PALABRAS CLAVE: centroides, distancia de Mahalanobis, gallinas.

*Universidad Central de Venezuela. Facultad de Agronomía. Instituto de Producción Animal Apartado postal 4579. Maracay 2101, galindez70@yahoo.com

${ }^{* *}$ Universidad Nacional Experimental Rómulo Gallegos, San Juan de los Morros. 
REVISTA DE LA UNIVERSIDAD DEL ZULIA. $3^{a}$ época. Año 11 № 29, 2020

Rafael Galíndez et al./// Diversidad fenotípica de aves criollas de postura...412-427

\section{Phenotypic diversity of native laying birds based on zoomometric characters}

ABSTRACT

In order to analyze the phenotypic diversity of Venezuelan Creole bird layers of the Laboratorio Sección de Aves, Instituto de Producción Animal, Facultad de Agronomía, Universidad Central de Venezuela, zoometric measures were taken of 220 male and female of the FAGRO ( $\mathrm{n}=33)$, GDB $(\mathrm{n}=65)$, IPA $(\mathrm{n}=36)$ and Maracay $(\mathrm{n}=86)$ breeds. The traits considered were live weight, thoracic perimeter, body length, thigh, leg, tarsus, wing, ear lobe, wattle, tail feathers, comb height and ear lobe width. Variance analysis were performed including sex and breed effects, comparing the average through Duncan test. Relationship coefficients of some body measurements with live weight were calculated. On the other hand, discriminant analyses and Mahalanobis distances were calculated, the centroids of every breed were plotted. All measurements were higher $(\mathrm{P}<0.05)$ in males with differences between 1 and 57\%. Differences were detected $(\mathrm{P}<0.05)$ between breeds, Maracay and IPA expressed higher values. Most correlation of the body measurements were highly significant, with higher magnitudes observed in IPA breed. Mahalanobis distances based in six discriminant trait, revealed that Maracay and FAGRO are the breeds more distances. It is concluded that there is high divergences between sexes moreover, it is detected high diversity between analyzed groups.

KEYWORDS: centroids, Mahalanobis distance, hens.

\section{Introducción}

Es imprescindible caracterizar los recursos genéticos de interés zootécnico disponibles antes de diseñar y ejecutar cualquier programa de conservación o mejora genética de las poblaciones locales de animales. En este sentido, la descripción de estos recursos se ejecuta compilando tres tipos de información: histórica, fenotípica y genética (FAO, 2012). Asimismo, se ha establecido que la caracterización fenotípica es fundamental para definir el inventario de individuos pertenecientes a las razas, líneas o grupos genéticos presentes en una determinada región, condición medioambiental o manejo específico (FAO, 2012).

Para el caso de las gallinas criollas venezolanas, si bien es cierto, que se han recabado muchos datos de caracteres productivos y funcionales (Galíndez et al., 2012; 
REVISTA DE LA UNIVERSIDAD DEL ZULIA. $3^{a}$ época. Año 11 N 29, 2020

Rafael Galíndez et al./// Diversidad fenotípica de aves criollas de postura...412-427

2014; Galíndez y Blanco, 2017), son pocos los estudios que se han realizado para dilucidar la diversidad morfométrica y genética de las poblaciones. Dentro de los estudios relacionados a la diversidad fenotípica, se ha explorado un poco con el análisis de los caracteres cualitativos (color del plumaje, orejilla, tarsos, tipo de cresta, etc.), los cuales son muy eficientes para definir las razas (Lucas y Galíndez, 2017). Por otro lado, no se han estudiado los rasgos zoométricos que, en algunos casos, no son suficientes para delimitar las razas, teniendo que recurrir a menudo al auxilio de caracteres productivos, cualitativos o el análisis genético molecular para separar con precisión los individuos de una raza, línea o grupo poblacional de los otros. En este orden de ideas, el uso de herramientas estadísticas como el análisis discriminante paso a paso ofrece un enorme potencial para definir las características que separan a las poblaciones, y clasificar por supuesto, los grupos de individuos.

El objetivo del presente trabajo fue estudiar la diversidad fenotípica de aves criollas venezolanas de postura basada en caracteres zoométricos.

\section{Materiales y Métodos}

Los datos se recolectaron de los ejemplares de gallos y gallinas presentes en el Laboratorio Sección de Aves del Instituto de Producción Animal de la Facultad de Agronomía de la Universidad Central de Venezuela (Laboratorio Sección de Aves), el cual tiene una altitud de 455 m.s.n.m, precipitación promedio de $1135 \mathrm{~mm}$, temperatura promedio de $25,5{ }^{\circ} \mathrm{C}$ y humedad relativa promedio de $74,0 \%$ (INIA, 2019).

Las aves estaban ubicadas en el galpón "A", que posee piso de concreto, tiene $64 \mathrm{~m}$ de largo, $6 \mathrm{~m}$ de ancho, altura mínima de 2,7 m y máxima de 5,0 m. Está dividido en 60 corrales con dimensiones de 2,0 $\mathrm{m}$ de largo, 2,0 $\mathrm{m}$ de ancho y 2,0 $\mathrm{m}$ de alto $(0,43 \mathrm{~m}$ de pared de concreto y $1,57 \mathrm{~m}$ de malla metálica). El piso de cada corral estaba cubierto de concha de arroz, los bebederos eran de tipo campana (32) y en otros corrales de tipo copa (28). Los comederos eran tipo tolva con una capacidad de alimentación máxima de veinte (20) aves por cada uno.

Para medir el peso se utilizó un equipo electrónico marca Salter Brecknell, con capacidad máxima de $45 \mathrm{~kg}$ y apreciación de 50 g. Para cuantificar las longitudes se 
REVISTA DE LA UNIVERSIDAD DEL ZULIA. $3^{a}$ época. Año 11 N² 29, 2020

Rafael Galíndez et al./// Diversidad fenotípica de aves criollas de postura...412-427

hizo uso de un vernier marca INOX con una amplitud máxima de 15,1 cm (apreciación de $1 \mathrm{~mm}$ ) y una cinta métrica con una extensión máxima de $150 \mathrm{~cm}$.

Se tomaron medidas de 220 aves, conformados por gallinas y gallos adultos; distribuidos por raza de la siguiente manera:

FAGRO: 33.

GDB: 65 .

IPA: 36.

Maracay: 86.

Total: 220 .

La alimentación se basó en el suministró de balanceado comercial para ponedoras a razón de $100 \mathrm{~g}$ por animal, con las siguientes características: proteína cruda (16\%), fibra cruda (5\%), extracto etéreo (3\%), extracto libre de nitrógeno (50\%).

Se consideraron 14 caracteres cuantitativos: peso corporal, longitud corporal, muslo, pierna, tarso, ala, orejilla, barbilla, plumas de la cola; perímetro pectoral; ancho de ala, orejilla, barbilla y altura de cresta (Tabla 1).

Se realizaron análisis de varianza para comparar las medidas zoométricas entre sexo y razas, utilizando el programa estadístico SAS (Littell et al., 2002). El modelo usado fue el siguiente:

$\mathrm{Y}_{\mathrm{ijk}}=\mu+\operatorname{Sex}_{\mathrm{i}}+\mathrm{R}_{\mathrm{J}}+\varepsilon_{\mathrm{ijk}} ;$ donde:

$\mathrm{Y}_{\mathrm{ijk}}=$ medida zoométrica.

$\mu=$ media teórica de la población.

Sex $_{i}=$ efecto del sexo del ave (macho, hembra).

$\mathrm{R}_{\mathrm{j}}=$ efecto de raza (FAGRO, GDB, IPA, Maracay).

$\varepsilon_{\mathrm{ijk}}=$ residual con media "0" y varianza " $\sigma$ ", normal e independientemente distribuido.

En análisis preliminares se comprobó la ausencia de la interacción sexo*raza, por tal motivo se excluyó de los análisis finales. Para verificar la diferencia entre los promedios se realizó la prueba de medias de Duncan (Steel et al., 1997). Se calcularon los coeficientes de correlación para determinar posibles relaciones entre el peso vivo (PV), perímetro torácico y la longitud corporal, muslo, pierna y tarso. 
REVISTA DE LA UNIVERSIDAD DEL ZULIA. $3^{a}$ época. Año 11 N² 29, 2020

Rafael Galíndez et al./// Diversidad fenotípica de aves criollas de postura...412-427

Tabla 1. Descripción de mediciones corporales en aves criollas de postura/Description of body measurements in Creole birds of lay

Longitud corporal

Distancia entre la punta del pico y la base de la cola (sin las plumas).

\begin{tabular}{|c|c|}
\hline $\begin{array}{l}\text { Perímetro } \\
\text { torácico }\end{array}$ & $\begin{array}{l}\text { La circunferencia del pecho tomada hasta la punta posterior de la } \\
\text { pechuga }\end{array}$ \\
\hline $\begin{array}{l}\text { Longitud } \\
\text { del muslo }\end{array}$ & $\begin{array}{l}\text { Distancia entre la articulación coxofemoral a la articulación de la } \\
\text { rodilla }\end{array}$ \\
\hline $\begin{array}{l}\text { Longitud de } \\
\text { la pierna }\end{array}$ & $\begin{array}{l}\text { Distancia entre la articulación de la rodilla hasta la articulación del } \\
\text { tarso }\end{array}$ \\
\hline $\begin{array}{l}\text { Longitud } \\
\text { del tarso }\end{array}$ & Distancia entre la articulación del taso y el origen del cuarto dedo \\
\hline $\begin{array}{l}\text { Longitud } \\
\text { del ala }\end{array}$ & $\begin{array}{l}\text { Distancia medida desde la unión del húmero con las vértebras } \\
\text { dorsales hasta la punta de las plumas primarias (el ala debe estar } \\
\text { extendida). }\end{array}$ \\
\hline $\begin{array}{l}\text { Ancho } \\
\text { del ala }\end{array}$ & $\begin{array}{l}\text { Longitud medida entre la unión del húmero con el cubito hasta la } \\
\text { última pluma secundaria (el ala debe estar extendida). }\end{array}$ \\
\hline $\begin{array}{l}\text { Altura de } \\
\text { la cresta }\end{array}$ & $\begin{array}{l}\text { Distancia entre la sierra más alta a la inserción de la cresta en el } \\
\text { cráneo }\end{array}$ \\
\hline $\begin{array}{l}\text { Longitud } \\
\text { de orejilla }\end{array}$ & Distancia entre la parte superior y la inferior de la orejilla. \\
\hline $\begin{array}{l}\text { Ancho } \\
\text { de orejilla }\end{array}$ & Longitud entre la parte rostral y la caudal de la orejilla. \\
\hline $\begin{array}{l}\text { Longitud } \\
\text { de barbilla }\end{array}$ & Distancia desde la base de la barbilla hasta el final de la misma. \\
\hline $\begin{array}{l}\text { Ancho } \\
\text { de barbilla }\end{array}$ & $\begin{array}{l}\text { Longitud entre la parte rostral y caudal de la barbilla (sin estar } \\
\text { extendida). }\end{array}$ \\
\hline $\begin{array}{l}\text { Longitud de } \\
\text { plumas de } \\
\text { cola }\end{array}$ & $\begin{array}{l}\text { Distancia desde la base del pigostilo (hueso de cola) hasta el final de } \\
\text { la misma. }\end{array}$ \\
\hline
\end{tabular}

Fuente: Gutiérrez, 2013; Pérez et al., 2004. 
REVISTA DE LA UNIVERSIDAD DEL ZULIA. $3^{a}$ época. Año 11 N 29, 2020

Rafael Galíndez et al./// Diversidad fenotípica de aves criollas de postura...412-427

Se realizó un análisis discriminante por pasos (stepwise) con el programa estadística SAS, con la finalidad de determinar las variables que serían usadas en el análisis de agrupamiento, este procedimiento establece las variables que tienen más poder discriminante (Littell et al., 2002).

Finalmente, se computaron las distancias de Mahalanobis entre razas y la representación gráfica de las mismas usando el complemento para Microsoft Excel XLSTAT (XLSTAT, 2018).

\section{Resultados y discusión}

La Tabla 2 muestra los promedios por sexo para gallinas y gallos criollos del Laboratorio Sección de Aves (LSA). Todas las variables consideradas resultaron superiores $(\mathrm{P}<0,05)$ en los machos, con diferencias que oscilan entre 1\% (ancho de orejilla) y $57 \%$ (longitud de barbilla).

Las diferencias entre sexo en cuanto a medidas zoométricas ha sido reportado con anterioridad (Lázaro et al., 2012; Cabarles, 2013; Guni et al., 2013; Sanmartin, 2014; Chincoya et al., 2016;), comentado los autores que las divergencias entre machos y hembras obedecen al dimorfismo sexual producto del control que ejercen algunas hormonas sobre estos caracteres. Asimismo, algunos investigadores asocian la mayor longitud de la cresta y barbilla de los machos con el desarrollo de las gónadas y la secreción de hormonas sexuales (Duguma, 2006; Bembide et al., 2013).

De manera general, los caracteres medidos tanto en machos como hembras superan algunos reportes de la literatura (Duguma, 2006; Chincoya et al., 2016); sin embargo, son inferiores a otro trabajo publicado (Bembide et al., 2013), pudiendo aseverar que las diferencias genéticas entre los grupos de aves del presente estudio y los trabajos citados se expresa en los valores fenotípicos mencionados, como consecuencia de los procesos de selección artificial aplicados en estas poblaciones, de la adaptación de los individuos a las condiciones ambientales, edad y estado fisiológico (Bembide et al., 2013).

Por otra parte, se ha mencionado que el dimorfismo sexual en esta especie está relacionado a altos niveles de poligamia social, que a su vez es consecuencia de la 
REVISTA DE LA UNIVERSIDAD DEL ZULIA. $3^{a}$ época. Año 11 N² 29, 2020

Rafael Galíndez et al./// Diversidad fenotípica de aves criollas de postura...412-427

competencia de los machos para acceder al apareamiento y la selección artificial dirigida hacia animales más grandes, pesados y con caracteres secundarios (tamaño de la cresta, orejillas y barbillas) muy bien definidos (Alabi et al., 2012).

Tabla 2. Medidas zoométricas para machos y hembras de cuatro razas de gallinas criollas/Zoometric measurements for male and female of four Creole hens breeds.

\begin{tabular}{|c|c|c|c|c|}
\hline \multirow{2}{*}{ Variable } & \multicolumn{2}{|c|}{ Promedio } & \multirow{2}{*}{$\begin{array}{c}\text { Desviación } \\
\text { estándar }\end{array}$} & \multirow{2}{*}{$\begin{array}{l}\text { Coeficiente } \\
\text { de } \\
\text { variación }\end{array}$} \\
\hline & Macho & Hembra & & \\
\hline Peso vivo (g) & $2595,0 \mathrm{a}$ & $1800,5 \mathrm{~b}$ & 408,4 & 21,4 \\
\hline Longitud corporal (cm) & $44,8 \mathrm{a}$ & $39,5 \mathrm{~b}$ & 3,4 & 8,3 \\
\hline Perímetro pectoral (cm) & $37,8 \mathrm{a}$ & $30,9 \mathrm{~b}$ & 4,2 & 13,6 \\
\hline Longitud de muslo $(\mathrm{cm})$ & $10,5 \mathrm{a}$ & $8,9 \mathrm{~b}$ & 0,9 & 10,4 \\
\hline Longitud de pierna $(\mathrm{cm})$ & $14,7 \mathrm{a}$ & $12,4 \mathrm{~b}$ & 1,3 & 9,6 \\
\hline Longitud de tarso $(\mathrm{cm})$ & $9,6 \mathrm{a}$ & $7,9 \mathrm{~b}$ & 1,1 & 12,3 \\
\hline Longitud de ala $(\mathrm{cm})$ & $38,9 a$ & $34,0 \mathrm{~b}$ & 3,5 & 9,7 \\
\hline Ancho de ala $(\mathrm{cm})$ & $20,1 \mathrm{a}$ & $17,5 \mathrm{~b}$ & 2,9 & 15,7 \\
\hline Altura de cresta $(\mathrm{cm})$ & $6,4 \mathrm{a}$ & $3,4 \mathrm{~b}$ & 1,6 & 39,5 \\
\hline Longitud de orejilla & & & 0,7 & 32,6 \\
\hline$(\mathrm{cm})$ & $3,5 \mathrm{a}$ & $2,1 \mathrm{~b}$ & & \\
\hline Ancho de orejilla $(\mathrm{cm})$ & $1,6 \mathrm{a}$ & $1,1 \mathrm{~b}$ & 0,15 & 15,2 \\
\hline Longitud de barbilla & & & 1,6 & 42,1 \\
\hline$(\mathrm{cm})$ & $6,5 \mathrm{a}$ & $2,8 \mathrm{~b}$ & & \\
\hline Ancho de barbilla (cm) & $3,8 \mathrm{a}$ & $1,7 \mathrm{~b}$ & 0,7 & 34,7 \\
\hline
\end{tabular}

Letras diferentes en la misma fila reflejan diferencias $(\mathrm{P}<0,05)$.

Con excepción de la longitud corporal (Tabla 2) todas las demás variables tienen coeficiente de variación superior al 9\%, por tanto se está en presencia de elevada variabilidad, situación que es descrita en la literatura como: una población sin uniformidad para los caracteres considerados (Matola, 2016), lo cual resultaría lógico, puesto que el grupo de animales evaluados incluye varias razas, por tanto, es probable 
REVISTA DE LA UNIVERSIDAD DEL ZULIA. $3^{a}$ época. Año 11 N 29, 2020 Rafael Galíndez et al./// Diversidad fenotípica de aves criollas de postura...412-427 que existan sub-divisiones por ésta causa. La aseveración anterior se corrobora con los resultados presentados en la Tabla 3 , en la cual se aprecian diferencias $(\mathrm{P}<0,05)$ en magnitudes entre razas para todos los caracteres medidos.

Diferencias entre razas, líneas o grupos han sido reportadas (Duguma, 2006; Al Atiyat, 2009; Galíndez et al., 2012; Cabarles, 2013; Galíndez et al., 2014), tanto para caracteres zoométricos como para rasgos productivos, coincidiendo los autores que las divergencias observadas tienen su origen en el genoma diferencial de las aves. De manera general, las razas Maracay e IPA expresaron mayores $(\mathrm{P}<0,05)$ valores para los rasgos morfométricos con muy pocas diferencias entre ellas (longitud corporal, ancho de ala, altura de cresta, longitud de orejilla, ancho y longitud de barbilla) (Tabla 3).

En promedio las razas mencionadas superan a las restantes en el peso corporal en un poco más de 290 g; estos valores, combinados con las longitudes corporales, muslo, pierna y tarso demuestran que las dos primeras razas son de mayor tamaño que las razas FAGRO y GDB.

Las similitudes entre las últimas razas mencionadas sugieren que pudiesen existir ancestros comunes; sin embargo y como se ha evidenciado, es necesario realizar estudios a nivel molecular para estudiar la diversidad de las poblaciones (Alabi et al., 2012).

En otro orden de ideas y a pesar que las condiciones de explotación, edad de los animales, clima, etc., son disímiles entre esta investigación y otros trabajos, algunas comparaciones pudiesen establecerse. Por ejemplo, el peso adulto de las razas Maracay e IPA es similar al de la raza española de gallinas Menorquina; mientras que las razas FAGRO y GDB son semejantes a la raza Mallorquina (Méndez, 2010). Asimismo, la longitud de las extremidades para este estudio es inferior a las razas españolas mencionadas, lo que supone que estas últimas son animales más altos, situación que responde a la selección dirigida hacia caracteres ornamentales realizada con anterioridad.

La Tabla 4 muestra los coeficientes de correlación y regresión lineal simple ente el peso vivo y las medidas morfométricas más importantes de gallos y gallinas del Laboratorio Sección de Aves. Sólo las correlaciones de peso vivo - longitud de pierna en GDB y peso vivo - longitud del tarso en FAGRO resultaron no significativas. Las 
REVISTA DE LA UNIVERSIDAD DEL ZULIA. $3^{a}$ época. Año 11 № 29, 2020

Rafael Galíndez et al./// Diversidad fenotípica de aves criollas de postura...412-427

correlaciones de menor magnitud se observaron en la raza FAGRO (peso vivo longitudes de muslo y pierna); mientras que las de mayor valor se evidenciaron en la raza IPA (peso vivo - longitud del muslo, corporal y pierna).

Tabla 3. Medidas zoométricas para varias razas de gallinas criollas venezolanas/Zoometric measurements for several breeds of Venezuelan Creole hens.

\begin{tabular}{lcccc} 
& \multicolumn{4}{c}{ Grupo Racial } \\
\cline { 2 - 5 } Variable & Maracay & IPA & FAGRO & GDB \\
\hline Peso vivo (g) & $2050,6 \mathrm{a}$ & $2005,8 \mathrm{a}$ & $1751,7 \mathrm{~b}$ & $1723,0 \mathrm{~b}$ \\
Longitud corporal (cm) & $41,3 \mathrm{a}$ & $40,0 \mathrm{~b}$ & $40,0 \mathrm{~b}$ & $38,7 \mathrm{c}$ \\
Perímetro pectoral (cm) & $32,1 \mathrm{~ns}$ & $32,0 \mathrm{~ns}$ & $31,9 \mathrm{~ns}$ & $31,1 \mathrm{~ns}$ \\
Longitud de muslo (cm) & $9,4 \mathrm{a}$ & $9,2 \mathrm{a}$ & $8,8 \mathrm{~b}$ & $8,8 \mathrm{~b}$ \\
Longitud de pierna (cm) & $13,0 \mathrm{a}$ & $13,1 \mathrm{a}$ & $12,5 \mathrm{~b}$ & $12,2 \mathrm{~b}$ \\
Longitud de tarso (cm) & $8,3 \mathrm{a}$ & $8,4 \mathrm{a}$ & $8,1 \mathrm{~b}$ & $7,7 \mathrm{c}$ \\
Longitud de ala (cm) & $35,1 \mathrm{a}$ & $35,8 \mathrm{a}$ & $33,8 \mathrm{~b}$ & $33,7 \mathrm{~b}$ \\
Ancho de ala (cm) & $18,8 \mathrm{a}$ & $17,7 \mathrm{~b}$ & $17,5 \mathrm{~b}$ & $16,4 \mathrm{c}$ \\
Altura de cresta (cm) & $3,6 \mathrm{c}$ & $4,5 \mathrm{a}$ & $4,1 \mathrm{~b}$ & $3,6 \mathrm{c}$ \\
Longitud de orejilla & $2,2 \mathrm{c}$ & $2,4 \mathrm{~b}$ & $2,6 \mathrm{a}$ & $2,2 \mathrm{c}$ \\
(cm) & & & & \\
Ancho de orejilla (cm) & $1,1 \mathrm{~b}$ & $1,2 \mathrm{a}$ & $1,1 \mathrm{~b}$ & $1,1 \mathrm{~b}$ \\
Longitud de barbilla & $3,2 \mathrm{~b}$ & $3,7 \mathrm{a}$ & $3,7 \mathrm{a}$ & $3,2 \mathrm{~b}$ \\
(cm) & & & & \\
Altura de barbilla (cm) & $1,9 \mathrm{~b}$ & $2,0 \mathrm{a}$ & $2,0 \mathrm{a}$ & $1,9 \mathrm{~b}$ \\
\hline Letras diferentes entre & filas expresan & diferencias $(\mathrm{P}<0,05)$, & $\mathrm{ns}: \mathrm{no}$ \\
significativo. & & & &
\end{tabular}

Las relaciones observadas coinciden con reportes anteriores (Pérez et al., 2004; Méndez, 2010; Méndez et al., 2011; Guni et al., 2013). Asimismo, en una caracterización de los fenotipos de las gallinas locales en Dekina, descubrieron correlaciones 
REVISTA DE LA UNIVERSIDAD DEL ZULIA. $3^{a}$ época. Año 11 N 29, 2020

Rafael Galíndez et al./// Diversidad fenotípica de aves criollas de postura...412-427

significativas para los parámetros peso vivo - largo corporal $(0,596)$, peso vivo perímetro pectoral $(0,704)$, peso vivo - largo de tarso (0,641) (Daikwo et al., 2011).

Los resultados sugieren que el aumento de cualquiera de estos parámetros corporales causará incremento en el peso del cuerpo, por tanto, pueden ser usadas cualquiera de estas medidas para estimar el peso corporal con elevada precisión (Alabi et al., 2012; Guni et al., 2013).

Por otra parte, estas relaciones resultan particularmente útiles en zonas, principalmente rurales, donde no existe la disponibilidad de equipos de medición del peso corporal (Alabi et al., 2012).

En la Tabla 5 se presentan los resultados del análisis discriminante paso a paso (stepwise) realizado con el programa estadístico SAS. De catorce variables originales, se seleccionaron seis, de acuerdo al procedimiento sugerido, estas son: altura de la cresta, longitud del muslo, longitud del tarso, longitud corporal, longitud de la barbilla y longitud del ala; el resto de las variables son retiradas del análisis, pues según el análisis discriminante, poco se diferencian las razas utilizando estas medidas (De la Fuente, 2011).

La variable que mejor discrimina es altura de la cresta, debido a que posee el mayor $\mathrm{R}^{2}$ parcial y el mayor valor de "F" (de la Fuente, 2011), el resto de las variables sigue el orden en el cual son mencionadas anteriormente. Cabe mencionar que el peso corporal no discrimina de manera eficiente las razas estudiadas, tal cual ha sido reportado para gallinas jordanas (Al - Atiyat, 2009). Sin embargo, largo corporal, largo del muslo, largo de tarso, largo de la barbilla y del ala son características que definen bien el tamaño de las gallinas (Chincoya et al., 2016).

Tomando como criterio la distancia de Mahalanobis, las razas más disímiles serían FAGRO y Maracay, seguidas de IPA - Maracay, luego GDB - Maracay, FAGRO - IPA, GDB - IPA y FAGRO - GDB (Tabla 6).

Las distancias de Mahalanobis son adimensionales, por tanto, mientras mayor sea el valor, la distancia entre los grupos es superior (Salas y Escobedo, 2008). En este orden de ideas, es lógico pensar que existen diferencias apreciables, no sólo morfológicas (color de plumaje, tarsos, etc.), sino en algunas medidas zoométricas 
REVISTA DE LA UNIVERSIDAD DEL ZULIA. $3^{a}$ época. Año 11 № 29, 2020

Rafael Galíndez et al./// Diversidad fenotípica de aves criollas de postura...412-427

entre las razas, lo que coincide con el análisis de varianza anterior, en el cual se observan algunas diferencias entre estas razas (Tabla 3).

Tabla 4. Coeficientes de correlación lineal simple entre el peso vivo (PV), longitud corporal (LC), perímetro pectoral (PP), longitud del muslo (LM), pierna (LP) y tarso (LT) en cuatro razas de gallinas criollas venezolanas/Simple lineal correlation coefficients between live weight (PV), body length (LC), pectoral perimeter (PP), thigh length (LM), leg (LP), and tarsus (LT) in four breeds of Venezuelan Creole hens.

\begin{tabular}{lcccc}
\cline { 2 - 5 } & \multicolumn{4}{c}{ Raza } \\
\cline { 2 - 5 } & Maracay & IPA & FAGRO & GDB \\
\hline Parámetros & \multicolumn{4}{c}{ Correlación } \\
\hline PV-LC & $0.66^{* *}$ & $0.70^{* *}$ & $0.49^{* *}$ & $0.42^{* *}$ \\
PV-PP & $0.57^{* *}$ & $0.59^{* *}$ & $0.62^{* *}$ & $0.67^{* *}$ \\
PV-LM & $0.68^{* *}$ & $0.83^{* *}$ & $0.39^{*}$ & $0.67^{* *}$ \\
PV-LP & $0.66^{* *}$ & $0.70^{* *}$ & $0.39^{*}$ & $0.2 \operatorname{lns}$ \\
PV-LT & $0.63^{* *}$ & $0.67^{* *}$ & $0.29 \mathrm{~ns}$ & $0.56^{* *}$ \\
\hline$* *(\mathrm{P}<0,01),{ }^{*}(\mathrm{P}<0,05)$, ns: no significativo &
\end{tabular}

Tabla 5. Análisis discriminante para los caracteres zoométricos de gallinas criollas venezolanas/Discriminant analyses for the zoometric characters of Venezuelan Creole hens.

\begin{tabular}{llccc}
\hline Paso & Variable introducida & $\mathrm{R}^{2}$ parcial & $\mathrm{F}$ & $\begin{array}{c}\lambda \mathrm{de} \\
\text { Wilks }\end{array}$ \\
\hline 1 & Longitud del muslo & 0,1305 & $10,81^{* *}$ & $0,8695^{* *}$ \\
2 & Altura de la cresta & 0,1849 & $16,26^{* *}$ & $0,7087^{* *}$ \\
3 & Longitud corporal & 0,0640 & $4,88^{* *}$ & $0,6633^{* *}$ \\
4 & Longitud del tarso & 0,0667 & $5,08^{* *}$ & $0,6190^{* *}$ \\
5 & Longitud de Barbilla & 0,0553 & $4,14^{* *}$ & $0,5848^{* *}$ \\
6 & Longitud del ala & 0,0528 & $3,92^{* *}$ & $0,5540^{* *}$ \\
\hline \hline
\end{tabular}

${ }^{* *} \mathrm{P}<0,01$ 
REVISTA DE LA UNIVERSIDAD DEL ZULIA. $3^{a}$ época. Año 11 № 29, 2020 Rafael Galíndez et al./// Diversidad fenotípica de aves criollas de postura...412-427

Las divergencias entre las razas estudiadas considerando algunos aspectos productivos (producción y peso de huevos) y reproductivos (fertilidad) han sido reportados en la literatura (Galíndez et al., 2012; 2014).

Tabla 6. Distancia de Mahalanobis para cuatro razas de gallinas criollas venezolanas/Mahalanobis distances for four breeds of Venezuelan Creole hens.

\begin{tabular}{lcccc}
\hline & FAGRO & GDB & IPA & MARACAY \\
\hline FAGRO & 0 & 0,948 & 1,592 & 3,194 \\
GDB & 0,948 & 0 & 1,285 & 1,821 \\
IPA & 1,592 & 1,285 & 0 & 2,038 \\
MARACAY & 3,194 & 1,821 & 2,038 & 0 \\
\hline
\end{tabular}

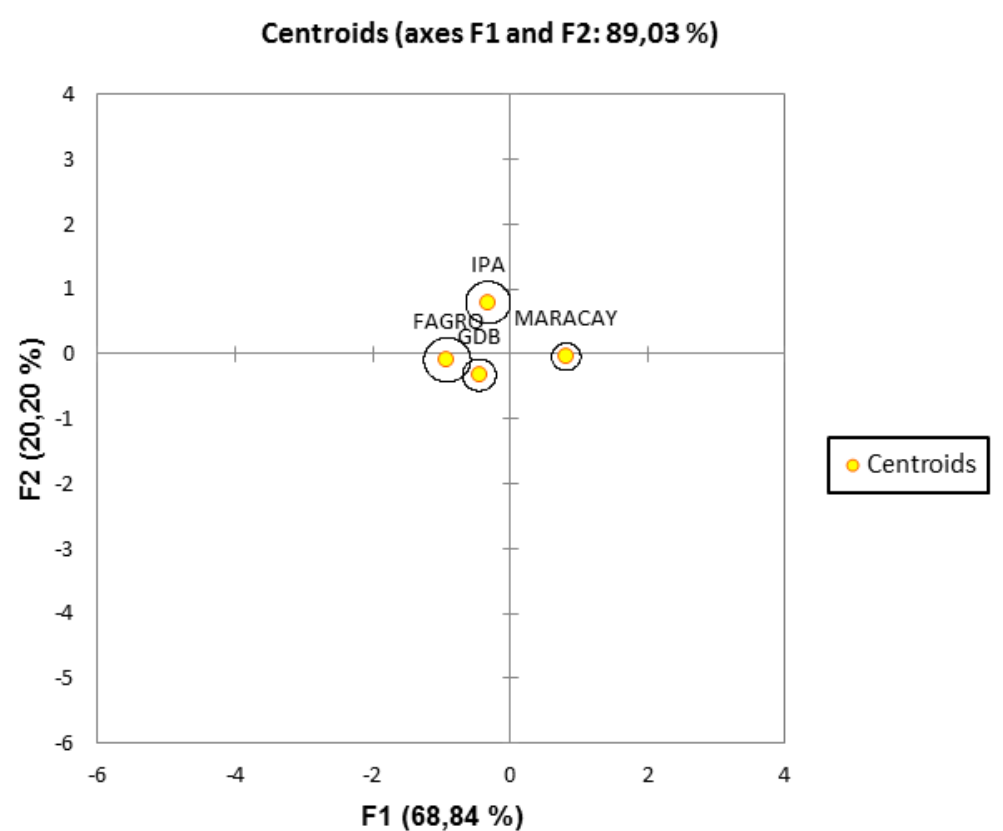

Figura 1. Representación gráfica de las distancias entre los centroides de cuatro razas de gallinas criollas venezolanas/Graphic representation of the distances between the centroids of four breeds of Venezuelan Creole hens. 
REVISTA DE LA UNIVERSIDAD DEL ZULIA. $3^{a}$ época. Año 11 N² 29, 2020 Rafael Galíndez et al./// Diversidad fenotípica de aves criollas de postura...412-427

La mayor distancia entre las razas FAGRO y Maracay es de esperar y se corresponde con la historia genealógica de estas razas, ya que, no comparten ancestros comunes (en el espacio de tiempo próximo que dio origen a las razas); asimismo, la cercanía detectada entre las razas GDB y FAGRO probablemente sea consecuencia de que los individuos de la primera raza son ancestros de la segunda (Galíndez, 2008). Aun así, se observa diversidad en los caracteres medidos entre las razas, lo cual representa una oportunidad para la conservación y mejora de los recursos zoogenéticos avícolas disponibles en Venezuela. Resultados homólogos han sido evidenciados (Abdelqader et al., 2008; Al - Atiyat, 2009) en poblaciones de gallinas criollas de Jordania, en las cuales, con la ayuda de la metodología del análisis discriminante se clasificaron exitosamente varios grupos genéticos de aves.

En la Figura 1 se observan de manera más clara las distancias entre las razas estudiadas. Los centroides para las razas FAGRO y GDB son graficados muy próximos, mientras que las razas IPA y Maracay son representadas más distantes de las dos anteriores, y estas a su vez, separadas entre sí.

A pesar de que algunos individuos mostraron rasgos morfométricos similares a una raza cuando pertenecen a otra, se pueden separar grandes grupos coincidentes con los promedios raciales (Figura 1), tendencia que es similar a lo señalado en estudios anteriores (FAO, 2009; Chincoya et al., 2016), en gallinas de Camboya y México, respectivamente, en los cuales lograron separar los grupos de gallinas, a pesar que entre un grupo y otro existieron similitudes para algunos caracteres, pero divergían en la mayoría de los otros rasgos zoométricos.

En este orden de ideas, se observa la diferenciación clara de la raza Maracay, mientras que las otras razas son mostradas con mayor cercanía. Tal resultado conduce a pensar que los caracteres a incluir en el análisis deben ser más determinantes o disímiles entre las razas.

\section{Conclusiones}

Las medidas zoométricas consideradas confirman la aseveración sobre el marcado dimorfismo sexual en estas aves, observando valores superiores para los machos. Se 
REVISTA DE LA UNIVERSIDAD DEL ZULIA. $3^{a}$ época. Año 11 N² 29, 2020

Rafael Galíndez et al./// Diversidad fenotípica de aves criollas de postura...412-427

detectó alta diversidad entre los grupos analizados, siendo ésta más pronunciada entre las razas Maracay y FAGRO. Por otra parte, a pesar que la herramienta de análisis discriminante es muy útil para clasificar las poblaciones animales, se recomienda que los caracteres a incluir en la investigación posean un alta poder de separación para obtener resultados satisfactorios.

\section{Referencias}

Abdelqader, A.; Wollny, C.; Gauly, M. (2008). On-farm investigation of local chicken biodiversity and performance potentials in rural areas of Jordan. AGRI 43: 49 - 58. 2008.

Alabi, O; Ngambi, J.; Norris, D.; Egena, S. (2012). Comparative study of three indigenous chicken breeds of South Africa: body weight and body linear measurements. Agricultural Journal 7(3): 220 - 225. 2012.

Al - Atiyat, R. (2009). Diversity of chicken populations in Jordan determined using discriminate analysis of performance traits. Int. J. Agric. Biol. 11: 373-380. 2009.

Bembide, C.; Hako, B.; Manjeli, Y.; Keambou, C. (2013). Caractérisation morphobiométrique de la poule locale en Centrafrique. Animal Genetic Resources 53: $33-44.2013$.

Cabarles, J. (2013). Phenotypic cluster and diversity analysis of native chickens in Western Visayas, Philippines. Animal Genetic Resources 53: 1-9. 2013.

Chincoya, H.; Jerez - Salas, M.; Herrera - Haro, G.; Mendoza - Nazar, P. (2016). Caracterización fenotípica y sistema de producción de las gallinas criollas en comunidades de Oaxaca. Revista Mexicana de Agroecosistemas 3: 87 - 98. 2016.

Daikwo, I.; Okpe, A.; Ocheja J. (2011). Phenotypic characterization of local chickens in Dekina. Int. J. Poult. Sci. 10: 444 - 447. 2011.

De La Fuente, S. (2011). Análisis discriminante. Facultad de Ciencias Económicas y Empresariales. Universidad Autónoma de Madrid. Madrid. España. 43 p. 2011.

Duguma, R. (2006). Phenotypic characterization of some indigenous chicken ecotypes of Ethiopia. LRRD 18:1 - 10. 2006.

FAO (2009). Characterization of indigenous chicken production systems in Cambodia. Prepared by M.T. Dinesh, E. Geerlings, J. Sölkner, S. Thea, O. Thieme and M. Wurzinger. AHBL - Promoting strategies for prevention and control of HPAI. Rome. 2009. 
REVISTA DE LA UNIVERSIDAD DEL ZULIA. $3^{a}$ época. Año 11 N² 29, 2020

Rafael Galíndez et al./// Diversidad fenotípica de aves criollas de postura...412-427

FAO (2012). Phenotypic characterization of animal genetic resources. FAO Animal Production and Health Guidelines No. 1l. Rome. 2012.

Galíndez, R. (2008). Fundamentos genéticos y de reproducción para el manejo de granjas avícolas con énfasis en gallinas ponedoras. Curso "Aspectos Básicos de la Producción de Gallinas Ponedoras". Facultad de Agronomía, Universidad Central de Venezuela. Maracay, Venezuela. CD-ROM. 2008.

Galíndez, R.; Peña, I.; Albarrán, A.; Prospert, J. (2012). Producción de huevos y fertilidad en cuatro líneas de gallinas reproductoras venezolanas. Rev Fac Agron (UCV) 38: 123 - 131. 2012.

Galíndez, R.; Peña, I.; Albarrán, A.; Prospert, J. (2014). Peso e indicadores de calidad interna del huevo de cuatro razas de gallinas reproductoras venezolanas. Zootecnia Trop 32: $207-215.2014$.

Galíndez, R.; Blanco, F. (2017). Eclosión, muerte embrionaria y calidad de pollitos en cuatro razas de gallinas reproductoras venezolanas. Revista Científica, FCV-LUZ XXVII: 56 - 61. 2017.

Guni, F.; Katule, A.; Mwakilembe, P. (2013). Characterization of local chickens in selected districts of the Southern Highlands of Tanzania: II. Production and Morphometric traits. LRRD 25, artículo \#11. 2013.

Gutiérrez, L. (2013). Caracterización morfológica de la gallina de traspatio del municipio de Pantepec, Chiapas. Tesis de Grado. Maestría en Ciencias en Producción Agropecuaria Tropical. Facultad de Medicina Veterinaria y Zootecnia. Universidad Autónoma De Chiapas. México. 55p. 2013.

INIA (2019). Unidad Agroclimatológica. Reporte de estación climatológica. Maracay, Aragua. Venezuela.

Lázaro, G.; Hernández, Z.; Vargas, L.; Martínez, L.; Pérez, A. (2012). Uso de caracteres morfométricos en la clasificación de gallinas locales. AICA 2: 109 - 114. 2012.

Littell, R.; Miliken, G.; Stroup, W.; Freud, R. (2002). SAS for Linear Models. $4^{\text {th }}$ edition. SAS Institute Inc. 633 p. 2002.

Lucas, G.; Galíndez, R. (2016). Rasgos fenotípicos cualitativos de aves criollas de postura en dos granjas experimentales venezolanas. Rev. Fac. Agron. UCV 43(2): 57 66.

Matola, M. (2016). Caracterización de las gallinas indígenas de Mozambique. Trabajo de grado, Doctorado. Departamento de Ciencia Animal y de los Alimentos. Universidad Autónoma de Barcelona. Barcelona, España. 197 p. 2016. 
REVISTA DE LA UNIVERSIDAD DEL ZULIA. $3^{a}$ época. Año 11 N² 29, 2020

Rafael Galíndez et al./// Diversidad fenotípica de aves criollas de postura...412-427

Méndez, Y. (2010). Zoometría comparada en las gallinas Baleares. Trabajo de Maestría. Máster Interuniversitario en Zootecnia y Gestión Sostenible: Ganadería Ecológica e Integrada Universidad de Córdoba. Córdoba, España. 36 p. 2010.

Méndez, Y.; Pons, A.; Francesch, A. (2011). Comparación de medidas zoométricas en las gallinas baleares. Arch. Zootec. 60: 445 - 448. 2011.

Pérez, A.; Polanco, G.; Pérez, Y. (2004). Algunas características morfológicas del exterior de la gallina local de la región central de la provincia de Villa Clara, Cuba. LRRD 16, Artículo \#10. 2004.

Salas, J.; Escobedo, M. (2008). Mahalanobis y las aplicaciones de su distancia estadística. Culcyt//Estadística 27: 13 - 20. 2008.

Sanmartin, L. (2014). Diagnóstico de los sistemas de crianza y caracterización morfológica de la gallina criolla (Gallus domesticus) en el Cantón Zapotillo Provincia de Loja. Tesis de grado. Universidad Nacional de Loja Área Agropecuaria y de Recursos Naturales Renovables Carrera de Medicina Veterinaria y Zootecnia. Loja, Ecuador. 129 p. 2014.

Steel, R.; Torrie, J.; Dickie, D. (1997). Principles and Procedures of Statistics a Biometrical Approach. Third edition. United State of America. McGraw - Hill. 666 p. 1997.

XLSTAT. XLSTAT Statistic Software, Free Edition. 2018. 